\title{
TITLE:
}

\section{Electronic excitations of fluoroethylenes}

AUTHOR(S):

Arulmozhiraja, S; Ehara, M; Nakatsuji, H

\section{CITATION:}

Arulmozhiraja, S ...[et al]. Electronic excitations of fluoroethylenes. JOURNAL OF CHEMICAL PHYSICS 2007, 126(4): 044306.

\section{ISSUE DATE:}

2007-01-28

URL:

http://hdl.handle.net/2433/50187

\section{RIGHT:}

Copyright 2007 American Institute of Physics. This article may be downloaded for personal use only. Any other use requires prior permission of the author and the American Institute of Physics. 


\title{
Electronic excitations of fluoroethylenes
}

\author{
Sundaram Arulmozhiraja and Masahiro Ehara \\ Department of Synthetic Chemistry and Biological Chemistry, Graduate School of Engineering, Kyoto \\ University, Katsura, Nishikyou-ku, Kyoto 615-8510, Japan \\ Hiroshi Nakatsujia) \\ Department of Synthetic Chemistry and Biological Chemistry, Graduate School of Engineering, Kyoto \\ University, Katsura, Nishikyou-ku, Kyoto 615-8510, Japan and Fukui Institute for Fundamental Chemistry, \\ Kyoto University, 34-4 Takano, Nishihiraki-cho, Sakyo-ku, Kyoto 606-8103, Japan
}

(Received 27 October 2006; accepted 4 December 2006; published online 25 January 2007)

\begin{abstract}
Several lowest-lying singlet electronic states of vinyl fluoride, trans-, cis-, and 1,1-difluoroethylene, trifluoroethylene, and tetrafluoroethylene were investigated by using symmetry-adapted cluster configuration interaction theory. Basis sets up to Dunning's aug-cc-pVTZ augmented with appropriate Rydberg functions were utilized for the calculations. Calculated excitation energies show a good agreement with the available experimental values. Even in the troublesome $\pi \rightarrow \pi^{*}$ transitions, the excitation energies obtained in the present study agree well with the experimental values except in one or two fluoroethylenes. Strong mixing between different states was noticed in a few fluoroethylenes; especially the mixing is very strong between $\pi-\pi^{*}$ and $\pi$-3p $\pi$ states in trifluoroethylene. No pure $\pi-\sigma^{*}$ excited state was found in almost all the fluoroethylenes. Several assignments and reassignments of features in the experimental spectra were suggested. The present study does not support the existing argument that the interaction between the $\pi-\pi^{*}$ and $\sigma$ - $\sigma^{*}$ states is the reason behind the blueshift of around $1.25 \mathrm{eV}$ in the $\pi-\pi^{*}$ excitation energy of tetrafluoroethylene. Possible reasons, including structural changes, for this shift are discussed in detail. Several low-lying triplet excited states were also studied. () 2007 American Institute of Physics. [DOI: 10.1063/1.2428296]
\end{abstract}

\section{INTRODUCTION}

Understanding the spectroscopy of haloethylenes is important for many reasons including for the fact that some of the haloethylenes are toxic pollutants. For this purpose, there were many studies in the past, especially experimental ones, on fluoroethylenes. ${ }^{1-6}$ However, even after many investigations by using UV spectroscopic and electron/ion-impact studies of these gaseous fluoroethylenes, the assignments and properties of the electronic states involved in the transitions are not free from ambiguity. For example, the second triplet state in vinyl fluoride found in an experimental study ${ }^{4}$ which is thought to be due to $\pi \rightarrow 3 s$ Rydberg excitation has been proposed to be due to $\pi \rightarrow \sigma^{*}$ valence excitation. ${ }^{7,8}$ However, a study later did not find any such excitation. ${ }^{5}$ Only tentative or speculative assignments regarding $\pi \rightarrow \sigma^{*}$ excitations in fluoroethylenes were made in most of the experimental studies but in some other studies no evidence was found for these valence-type excitations.

The absorption spectra of all the six fluoroethylenes were measured by Belanger and Sandorfy. ${ }^{1}$ They found that all the transitions of the fluoroethylenes down to $10.33 \mathrm{eV}$ are either valence-shell or Rydberg transitions of $\pi$ electrons. Lake and Thompson reported the photoelectron spectra of vinyl fluoride, 1,1-difluoroethylene, and tetrafluoroethylene. ${ }^{2}$

\footnotetext{
a) Author to whom correspondence should be addressed. Electronic mail: hiroshi@sbchem.kyoto-u.ac.jp
}

The electronic spectra of mono- to trifluoroethylene were studied by Dauber and Brith. ${ }^{3}$ The existence of the $\pi-\sigma^{*}$ state at lower energy than the $\pi-\pi^{*}$ state was verified for cis-difluoroethylene and trifluoroethylene. The $\pi \rightarrow \sigma^{*}$ absorption band was found to be very weak and was observed clearly in the solid phase and in $\mathrm{Kr}$ matrix. In gaseous difluoroethylene, however, it was not observed at all. ${ }^{3}$ Coggiola et al. ${ }^{4}$ investigated the electron-impact excitation spectra of all the fluoroethylenes. The second singlet $\rightarrow$ triplet transition was observed in vinyl fluoride with a maximum intensity at $6.4 \mathrm{eV}$. ${ }^{4}$ It was very weak in nature. The threshold electronimpact excitation spectra of vinyl fluoride and 1,1difluoroethylene were observed by Verhaart and Brongersma. ${ }^{5}$ The UV photoabsorption spectrum and electron energy loss spectra of tetrafluoroethylene have been reported recently by Eden et al. ${ }^{6}$ They, for the first time, proposed a Rydberg series of $n d \pi$ character. Robin reviewed the electronic spectra of haloethylenes including fluoroethylenes. ${ }^{7,8}$ An important fact is that high-level theoretical studies, which might help us to assign the transitions and eventually could help us to understand the electronic spectra, were not made in the past on the series of fluoroethylenes.

Photodissociation studies on fluoroethylenes were made in the past. ${ }^{9-18}$ The fluorinated ethylenes have very interesting chemical properties. One important example is that as the number of fluorine substitution increases, the double bond $\mathrm{C}=\mathrm{C}$ actually becomes weaker; this $\mathrm{C}=\mathrm{C}$ bond in tetrafluoroethylene is weaker than the typical $\mathrm{C}-\mathrm{C}$ single bond. Hence, different dissociation channels were found for differ- 
ent fluoroethylenes. These dissociation channels include atomic $\mathrm{H}$ and $\mathrm{F}$ eliminations, molecular $\mathrm{HF}, \mathrm{H}_{2}$, and $\mathrm{F}_{2}$ eliminations, and $\mathrm{C}=\mathrm{C}$ bond breaking. In most of the cases, the existing mechanisms behind the photolysis are either speculative or not clearly known due to the lack of theoretical studies. In addition to the expected involvement of various singlet states in the photodissociation mechanisms, the involvement of triplet states in the photolysis and photoisomerization of the difluoroethylenes was also evidenced in a few studies. ${ }^{9,19}$ Though complete studies on the photochemistry of the fluoroethylenes using theoretical calculations would explain the photochemical mechanisms fully, theoretical studies of the electronic spectra of fluoroethylenes could be a first step in this direction.

Apart from some uncertain assignments and the electronic state properties, there is an interesting fact about the fluoroethylenes. The vertical ionization potential (IP) of a $\pi$ electron in the $\mathrm{C}=\mathrm{C}$ double bond remains very nearly constant in the series from ethylene to tetrafluoroethylene. The $\sigma$ molecular orbitals (MOs) in the same series, however, are strongly stabilized on fluorination. This selective stabilization of the $\sigma$ MOs is called perfluoro effect. ${ }^{20}$ From the MO calculations it has been concluded that the insensitivity of the $\pi$-electron IPs is a consequence of the fact that the fluorination tends to stabilize the $\pi$ MO due to the high electronegativity of the fluorine atom, but this stabilization is countered by a strong $\mathrm{C}-\mathrm{F} \pi$ antibonding contribution which has the same magnitude as the electronegativity effect. On the other hand, these two effects work in the same direction for $\sigma$ MOs. Expectedly, $\pi \rightarrow \pi^{*}$ excitation energy is also seen to remain constant from ethylene to trifluoroethylene, but surprisingly the shift between trifluoroethylene to tetrafluoroethylene is around $1.25 \mathrm{eV}$ blueshift. This is a most interesting fact on fluoroethylenes. Explanations and counterexplanations were given in the past to support this observation; however, these explanations have not been tested properly.

For these above-mentioned reasons we studied the electronic spectra of the complete series of fluoroethylenes, vinyl fluoride, trans-, cis-, and 1,1-difluoroethylene, trifluoroethylene, and tetrafluoroethylene by using symmetry-adapted cluster $^{21} /$ configuration interaction ${ }^{22-24}$ (SAC-CI) theory. High-level theoretical studies were not made in the past on fluoroethylenes except a recent study on vinyl fluoride. ${ }^{25}$ Hence studying the electronic spectra of the whole series of the fluoroethylenes by using high-level theories would provide a good opportunity to fully understand the electronic structure of the fluoroethylenes and to draw a conclusion regarding the effects of fluorine substitution on excited states of ethylene.

The main focus of the present study is to obtain the electronic spectra of all the six fluoroethylenes and assign their valence as well as Rydberg transitions. This includes both singlet and triplet excitations. Special attention has been paid to understand the $\pi \rightarrow \pi^{*}$ excitation energy shift from trifluoroethylene to tetrafluoroethylene.

\section{COMPUTATIONAL DETAILS}

All computations were performed with GAUSSIAN03 suite of programs. ${ }^{26}$ The vertical absorption spectra of all the fluo- roethylenes were obtained using SAC-CI singles and doubles (SD)- $R$ method with LevelThree accuracy utilizing Dunning's valence triple-zeta basis set without $d$ polarization function for $\mathrm{H}$ atoms, hereafter cc-pVTZ $(-d)$, and Dunning's augmented valence triple-zeta basis sets, aug-cc-pVTZ, for C and $\mathrm{F}$ atoms. ${ }^{27}$ These basis sets were duly augmented with two sets of Rydberg spd functions for carbon $\left(\zeta_{s}=0.0437\right.$ and $0.01725, \zeta_{p}=0.0399$ and 0.01575 , and $\zeta_{d}=0.0285$ and $0.01125)$ and two sets for fluorine $\left(\zeta_{s}=0.0684\right.$ and 0.0270 , $\zeta_{p}=0.0551$ and 0.02175 , and $\zeta_{d}=0.0285$ and 0.01125$)$. This basis set combination provided excellent results for the excitations in vinyl chloride. ${ }^{28}$ In all the fluoroethylenes, the electronic spectra include possible singlet valence excitations and the first Rydberg series $(3 R \leftarrow N)$ involving transitions from highest occupied molecular orbital (HOMO), $\pi$ orbitals in this case, to Rydberg $3 s$ (one), $3 p$ (three), and $3 d$ (five) orbitals. Several low-lying triplet excitations in all the fluoroethylenes were also studied by using the same level of theory. In all the cases, we use " $\sigma$ " and " $\pi$ " to denote inplane and out-of-plane Rydberg orbitals ( $n p$ and $n d$ ), respectively. Vertical excitation energies for all the fluoroethylenes, except for the trifluoroethylene, were obtained at their respective experimental geometries found in the literature. Since the experimental geometry for the trifluoroethylene available in the literature seems not accurate, the vertical excitation energies for this fluoroethylene were calculated at B3LYP/aug-cc-pVTZ optimized geometry.

\section{RESULTS AND DISCUSSIONS}

\section{A. Vinyl fluoride}

Vinyl fluoride, the first member of the fluoroethylene family, has $C_{s}$ symmetry and the molecule was placed in the $x y$ plane for the electronic spectra calculations.

Recently, Barbatti et al. ${ }^{25}$ investigated the excited-state energy surfaces of vinyl fluoride by using multireference configuration interaction with singles and doubles (MRCISD). They also extensively studied the vertical spectrum of vinyl fluoride, including in total six valence and Rydberg excited states. They used different extended reference spaces with large basis sets for their calculations. See their original paper $^{25}$ for further computational details. Size-extensivity corrections were taken into account by means of the extended Davidson correction. The vertical spectrum was calculated also by means of resolution of the identity approximation coupled cluster singles and doubles (RI-CC2) method. So comparing the SAC-CI results obtained for the vinyl fluoride in the present study with those MRCI and RI-CC2 results is meaningful and would provide an idea about the SAC-CI results of fluoroethylenes.

Vertical singlet excitation energies and oscillator strengths calculated for the vinyl fluoride using SAC-CI were tabulated in Table I along with the available experimental and theoretical results. Table I shows that the main excitation in vinyl fluoride is $\pi \rightarrow \pi^{*}$ valence excitation. The excitation energy $(7.68 \mathrm{eV})$ calculated for this transition agrees very well with the available experimental values $(7.45$ and $7.50 \mathrm{eV})$. The MRCI/ $d^{\prime}$-aug-cc-pVDZ value $(7.86 \mathrm{eV})$ calculated for the same transition has around $0.4 \mathrm{eV}$ differ- 
TABLE I. Several lowest-lying singlet excited states of vinyl fluoride obtained using SAC-CI at experimental geometry (Ref. 29). Excitation energies ( $\Delta E$ ), oscillator strengths $(f)$, and second moments $\left(\left\langle r^{2}\right\rangle\right)$.

\begin{tabular}{|c|c|c|c|c|c|c|c|c|c|}
\hline State & Nature & $\Delta E(\mathrm{eV})$ & $f$ & $\left\langle r^{2}\right\rangle$ & $\mathrm{MCSCF}^{\mathrm{a}}$ & MR-CISD ${ }^{a}$ & $\mathrm{MR}-\mathrm{CISD}+\mathrm{Q}^{\mathrm{a}}$ & $\mathrm{CC} 2^{\mathrm{a}}$ & Expt. \\
\hline $1^{1} A^{\prime \prime}$ & $\pi-3 s$ & 7.11 & 0.0549 & 193.5 & 5.89 & 7.15 & 7.42 & 7.12 & $\begin{array}{l}6.98,{ }^{\mathrm{b}} 7.02, \\
7.04,{ }^{\mathrm{d}} 7.08^{\mathrm{d}}\end{array}$ \\
\hline $3^{1} A^{\prime \prime}$ & $\pi-3 p \sigma_{y}$ & 7.96 & 0.0027 & 202.3 & 6.72 & 7.98 & 8.26 & 7.99 & \\
\hline $3{ }^{1} A^{\prime}$ & $\pi-3 p \pi$ & 8.19 & 0.0281 & 215.4 & 6.82 & 8.17 & 8.37 & 8.51 & $809,{ }^{b} 8.08^{c}$ \\
\hline $4{ }^{1} A^{\prime \prime}$ & $\pi-3 d \sigma$ & 8.50 & 0.0016 & 213.8 & & & & & $8.59^{\mathrm{b}}$ \\
\hline $5^{1} A^{\prime \prime}$ & $\pi-3 d \sigma$ & 8.80 & 0.0110 & 250.8 & & & & & \\
\hline $6{ }^{1} A^{\prime \prime}$ & $\pi-3 d \sigma$ & 8.88 & 0.0264 & 253.2 & & & & & $8.87^{\mathrm{c}}$ \\
\hline $4{ }^{1} A^{\prime}$ & $\pi-3 d \pi$ & 8.92 & 0.0146 & 262.7 & & & & & \\
\hline
\end{tabular}

${ }^{\mathrm{a}}$ Reference 25 .

${ }^{\mathrm{b}}$ Reference 1.

${ }^{\mathrm{c}}$ Reference 4 .

${ }^{\mathrm{d}}$ Reference 5 .

ence with the experimental value. Even the inclusion of Davidson's size-extensivity correction did not improve the result. The only value close to the present result is the $\mathrm{CC} 2 /$ $d^{\prime}$-aug-cc-pVDZ value $(7.72 \mathrm{eV})$. But the $\pi$ - $3 p \pi$ excitation energy calculated using the same $\mathrm{CC} 2$ level $(8.51 \mathrm{eV})$ has around $0.4 \mathrm{eV}$ difference with the experimental values $(8.08$ and $8.09 \mathrm{eV})$, while the $\mathrm{SAC}-\mathrm{CI}$ result $(8.19 \mathrm{eV})$ once again agrees well with the experimental values. In all the other transitions too, the SAC-CI results agree very well with the available experimental results. Foregoing facts reveal clearly that highly reliable results can be obtained for fluoroethylenes by using SAC-CI theory with the selected basis set. Importantly, the results derived using SAC-CI are in better agreement with the experimental values than those derived using the other theoretical calculations. This gives the confidence that the SAC-CI theory with the selected basis set can be used to study all the other fluoroethylenes for which highlevel theoretical results are not available.

Table II shows the calculated triplet excitation energies for the vinyl fluoride with the existing experimental values. Triplet $\pi-\pi^{*}$ excitation energy calculated using SAC-CI agrees excellently with the experimental values. Coggiola $e t$ $a l .{ }^{4}$ found the second triplet state at $6.4 \mathrm{eV}$. They suggested that this might be due to triplet $\pi$-3s Rydberg excitation. Robin, ${ }^{7,8}$ however, argued that this transition at $6.4 \mathrm{eV}$ should not be due to a triplet $\pi-3 s$ Rydberg excitation because such an assignment makes the energy difference between this proposed triplet state and its respective singlet state around $0.6 \mathrm{eV}$, whereas 0.12 to $0.25 \mathrm{eV}$ energy difference is the normal range for Rydberg configurations. So he assumed that this $6.4 \mathrm{eV}$ triplet has the $\pi-\sigma^{*}$ configuration. But no excitation at $6.4 \mathrm{eV}$ was found in the study later by Verhaart and Brongersma. ${ }^{5}$ They restrained themselves from making any concrete conclusion regarding this due to their low resolution measurements; however, since there was clearly no direct excitation at an energy loss of $6.4 \mathrm{eV}$ for the impact energies between 6.5 and $9.5 \mathrm{eV}$, they concluded that such an excitation is doubtful. The present study firmly reveals that there is no triplet excitation at $6.4 \mathrm{eV}$. So we believe that the second triplet state found in the earlier study ${ }^{4}$ might be due to some contaminants. The $\pi-3 s$ triplet Rydberg excitation energy calculated using SAC-CI $(7.03 \mathrm{eV})$ agrees well with the experimental values $(6.85$ and $6.87 \mathrm{eV})$.

\section{B. trans-, cis-, and 1,1-difluoroethylenes}

Electronic-state calculations for the transdifluoroethylene were done within $C_{2 h}$ symmetry, with the molecule placed in the $x y$ plane. Table III shows the vertical singlet excitation energies with oscillator strengths calculated using SAC-CI with the available experimental values. The excitation energy $(7.79 \mathrm{eV})$ obtained for the $\pi \rightarrow \pi^{*}$ transition, the main excitation with the largest oscillator strength, agrees well with the experimental values $(7.56$ and $7.45 \mathrm{eV})$. But at the same time, an energy difference of around $0.4 \mathrm{eV}$ was noticed for the $\pi \rightarrow 3 s$ excitation between the SAC-CI

TABLE II. Several lowest-lying triplet excited states of vinyl fluoride obtained using SAC-CI at experimental geometry (Ref. 29). Excitation energies $(\Delta E)$.

\begin{tabular}{lccc}
\hline \hline State & Nature & $\begin{array}{c}\text { SAC-CI } \\
\Delta E(\mathrm{eV})\end{array}$ & Expt. \\
\hline $1^{3} A^{\prime}$ & $\pi-\pi^{*}$ & 4.39 & $4.40,{ }^{\mathrm{a}} 4.50^{\mathrm{b}}$ \\
& $\pi-3 s ?$ (or) $\pi-\sigma^{*} ?$ & & $6.40^{\mathrm{a}}$ \\
$1{ }^{3} A^{\prime \prime}$ & $\pi-3 s$ & 7.03 & $6.85,{ }^{\mathrm{b}} 6.87^{\mathrm{b}}$ \\
$2^{3} A^{\prime \prime}$ & $\pi-3 p \sigma_{x}$ & 7.72 & \\
$3^{3} A^{\prime \prime}$ & $\pi-3 p \sigma_{y}$ & 7.92 & \\
$2^{3} A^{\prime}$ & $\pi-3 p \pi$ & 8.01 & \\
$4^{3} A^{\prime \prime}$ & $\pi-3 d \sigma$ & 8.43 & \\
$5^{3} A^{\prime \prime}$ & $\pi-3 d \sigma$ & 8.77 & \\
$3^{3} A^{\prime}$ & $\pi-3 d \pi$ & 8.91 & \\
$4^{3} A^{\prime}$ & $\pi-3 d \pi$ & 9.00 & \\
\hline \hline
\end{tabular}

${ }^{\mathrm{a}}$ Reference 4.

${ }^{\mathrm{b}}$ Reference 5 . 
TABLE III. Several lowest-lying singlet excited states of trans-difluoroethylene obtained using SAC-CI at experimental geometry (Ref. 30). Excitation energies $(\Delta E)$, oscillator strengths $(f)$, and second moments $\left(\left\langle r^{2}\right\rangle\right)$.

\begin{tabular}{lccccc}
\hline \hline & & \multicolumn{3}{c}{ SAC-CI } \\
\cline { 3 - 5 } State & Nature & $\Delta E(\mathrm{eV})$ & $f$ & $\left\langle r^{2}\right\rangle$ & \multirow{2}{*}{ Expt. } \\
\hline $1{ }^{1} A_{g}$ & G. S. & & & 287.9 & \\
$1{ }^{1} B_{g}$ & $\pi-\sigma^{*} / 3 p \sigma_{x}$ & 7.02 & $\ldots$ & 303.7 & \\
$1{ }^{1} A_{u}$ & $\pi-3 s$ & 7.40 & 0.0438 & 330.1 & $6.79,{ }^{\mathrm{a}} 6.99,{ }^{\mathrm{b}} 6.44^{\mathrm{c}}$ \\
$2{ }^{1} B_{g}$ & $\pi-3 p \sigma_{y}$ & 7.77 & $\ldots$ & 333.1 & $7.88^{\mathrm{c}}$ \\
$1{ }^{1} B_{u}$ & $\pi-\pi^{*}$ & 7.79 & 0.3197 & 299.2 & $756,{ }^{\mathrm{a}} 7.45,{ }^{\mathrm{b}} 7.39^{\mathrm{c}}$ \\
$2{ }^{1} A_{g}$ & $\pi-3 p \pi$ & 8.17 & $\ldots$ & 350.8 & $8.25^{\mathrm{a}, \mathrm{b}}$ \\
$2{ }^{1} A_{u}$ & $\pi-3 d \sigma$ & 8.73 & 0.0261 & 359.9 & \\
$3{ }^{1} A_{u}$ & $\pi-3 d \sigma$ & 8.97 & 0.0037 & 399.6 & \\
$4{ }^{1} A_{u}$ & $\pi-3 d \sigma$ & 9.07 & 0.0166 & 423.6 & \\
$2{ }^{1} B_{u}$ & $\pi-3 d \pi$ & 9.07 & 0.0006 & 415.8 & \\
$3{ }^{1} B_{u}$ & $\pi-3 d \pi$ & 9.20 & 0.0470 & 435.5 & $8.82,{ }^{\mathrm{b}} 8.80^{\mathrm{c}}$ \\
\hline \hline
\end{tabular}

${ }^{\mathrm{a}}$ Reference 7.

${ }^{\mathrm{b}}$ Reference 1.

${ }^{\mathrm{c}}$ Reference 4.

and the experimental values. It should be mentioned here that for this particular transition, there is no unanimity among the available experimental values. The $\pi$ - $3 s$ state has some moderate oscillator strength while $\pi \rightarrow 3 p$ transition is $u \leftrightarrow u$ forbidden for this centrosymmetric fluoroethylene. The first excited state, $\pi-3 p \sigma_{x}$, has contributions from optically forbidden $\pi \rightarrow \sigma^{*}$ transition. So the excitation obtained at $7.02 \mathrm{eV}$ can be assigned due to the mixing of these two configurations. Robin ${ }^{7}$ mentioned that apart from $\pi \rightarrow 3 s$, $\pi \rightarrow 3 p$, and $\pi \rightarrow \pi^{*}$ excitations, a band may present at $7.07 \mathrm{eV}$ in trans-fluoroethylene. He tentatively assigned this band as due to $\pi \rightarrow \sigma^{*}$ transition. On the other hand, the study by Dauber and Brith ${ }^{3}$ did not find any such transition. The present study supports the assignment of $\pi \rightarrow \sigma^{*}$ transition. Triplet excitation energies calculated for several states were tabulated in Table IV. Only one experimental value, for the triplet $\pi-\pi^{*}$ excitation, is available for comparison. The energy difference of about $0.2 \mathrm{eV}$ was seen between SAC-CI and experimental values.

cis-difluoroethylene has $C_{2 v}$ symmetry. The electronic spectrum calculation was done within this symmetry, with the molecule placed in the $y z$ plane, where the $y$ axis is the

TABLE IV. Several lowest-lying triplet excited states of transdifluoroethylene obtained using SAC-CI at experimental geometry (Ref. 30). Excitation energies $(\Delta E)$.

\begin{tabular}{cccc}
\hline \hline State & Nature & $\begin{array}{c}\text { SAC-CI } \\
\Delta E(\mathrm{eV})\end{array}$ & Expt. \\
\hline $1{ }^{3} B_{u}$ & $\pi-\pi^{*}$ & 4.40 & $4.18^{\mathrm{a}}$ \\
$1{ }^{3} B_{g}$ & $\pi-\sigma^{*} / 3 p \sigma_{x}$ & 6.76 & \\
$1{ }^{3} A_{u}$ & $\pi-3 s$ & 7.31 & \\
$2{ }^{3} B_{g}$ & $\pi-3 p \sigma_{y}$ & 7.72 & \\
$1{ }^{3} A_{g}$ & $\pi-3 p \pi$ & 8.07 & \\
$2{ }^{3} A_{u}$ & $\pi-3 d \sigma$ & 8.62 & \\
$3{ }^{3} A_{u}$ & $\pi-3 d \sigma$ & 8.99 & \\
$2{ }^{3} B_{u}$ & $\pi-3 d \pi$ & 9.01 & \\
$3{ }^{3} B_{u}$ & $\pi-3 d \pi$ & 9.08 & \\
\hline
\end{tabular}

${ }^{\mathrm{a}}$ Reference 4 . molecular axis. The calculated results were tabulated in Table $\mathrm{V}$ with the available experimental values. The excitation energy for the $\pi-\pi^{*}$ state calculated using SAC-CI agrees with the experimental values-the difference of about $0.3 \mathrm{eV}$ is noticed at the maximum. A valence excitation at $6.84 \mathrm{eV}$ which appeared in an experimental study ${ }^{3}$ was considered as an evidence for an earlier assignment to $\pi \rightarrow \sigma^{*}$ valence excitation. ${ }^{7,8}$ However, it should be noticed that Dauber and Brith ${ }^{3}$ observed this transition, at $6.84 \mathrm{eV}$, only in the solid phase. In gaseous difluoroethylene it was not observed at all. The present study does not show any excitation near $6.84 \mathrm{eV}$. On the other hand the $\pi-3 p \sigma_{z}$ state has some contribution from $\pi$ - $\sigma^{*}$ configuration. Experimental results show that the $\pi-\pi^{*}$ excitation energy in cis- is around $0.3 \mathrm{eV}$ larger than that in trans-difluoroethylene. Expectedly, the same trend was also obtained in the SAC-CI calculations. The larger $\pi-\pi^{*}$ excitation energy in cis-difluoroethylene is due to the fact that in this fluoroethylene both fluorine atoms are in cis position yielding more antibonding character of $\pi^{*}$ which resulted in higher excitation energy. The vertical triplet excitation energies obtained for several states of cisdifluoroethylene were tabulated in Table VI. Again, the SAC-CI $\pi-\pi^{*}$ excitation energy $(4.55 \mathrm{eV})$ agrees very well with the experimental value $(4.43 \mathrm{eV})$.

1,1-difluoroethylene has $C_{2 v}$ symmetry so like in cisdifluoroethylene, all the excitations except the excitations with $A_{2}$ symmetry are allowed. The molecule was kept in the $y z$ axis with the $z$ axis as the molecular axis for the calculations. The calculated vertical singlet excitation energies with oscillator strengths along with the available experimental values were summarized in Table VII. The triplet excitation energies were tabulated in Table VIII.

Table VII shows that the SAC-CI values are in good agreement with the experimental values. The $\pi$ - $\pi^{*}$ excited state has some contribution from $\pi-3 p \pi$ configuration. Of many excited states, the $\pi-3 p \pi$ state needs special attention. The oscillator strength calculated for this transition is higher than those obtained for the respective transitions in other two 
TABLE V. Several lowest-lying singlet excited states of cis-difluoroethylene obtained using SAC-CI at experimental geometry (Ref. 31). Excitation energies $(\Delta E)$, oscillator strengths $(f)$, and second moments $\left(\left\langle r^{2}\right\rangle\right)$.

\begin{tabular}{|c|c|c|c|c|c|}
\hline \multirow[b]{2}{*}{ State } & \multirow[b]{2}{*}{ Nature } & \multicolumn{3}{|c|}{ SAC-CI } & \multirow[b]{2}{*}{ Expt. } \\
\hline & & $\Delta E(\mathrm{eV})$ & $f$ & $\left\langle r^{2}\right\rangle$ & \\
\hline $1^{1} A_{1}$ & G. S. & & & 249.9 & \\
\hline $1{ }^{1} B_{1}$ & $\pi-3 s$ & 6.68 & 0.0380 & 271.3 & $6.49,^{\mathrm{a}, \mathrm{b}} 6.52^{\mathrm{c}}$ \\
\hline $2{ }^{1} B_{1}$ & $\pi-3 p \sigma_{z} / \sigma^{*}$ & 7.37 & 0.0027 & 288.3 & \\
\hline $1{ }^{1} A_{2}$ & $\pi-3 p \sigma_{y}$ & 8.02 & $\ldots$ & 318.2 & \\
\hline $2{ }^{1} A_{1}$ & $\pi-3 p \pi$ & 8.09 & 0.0111 & 314.3 & $7.94,{ }^{\mathrm{a}} 7.82,{ }^{\mathrm{b}} 8.38^{\mathrm{c}}$ \\
\hline${ }^{1} B_{2}$ & $\pi-\pi^{*}$ & 8.12 & 0.3825 & 265.1 & $7.81,{ }^{\mathrm{a}} 7.82^{\mathrm{b}, \mathrm{c}}$ \\
\hline $2{ }^{1} A_{2}$ & $\pi-3 d \sigma$ & 8.88 & $\ldots$ & 355.3 & \\
\hline $4{ }^{1} B_{1}$ & $\pi-3 d \sigma$ & 8.92 & 0.0365 & 366.2 & \\
\hline $3{ }^{1} A_{1}$ & $\pi-3 d \pi$ & 8.96 & 0.0058 & 363.4 & \\
\hline $5{ }^{1} B_{1}$ & $\pi-3 d \sigma$ & 8.99 & 0.0008 & 376.1 & \\
\hline $2{ }^{1} B_{2}$ & $\pi-3 d \pi$ & 9.12 & 0.0591 & 392.9 & $8.80,^{b} 9.01^{c}$ \\
\hline
\end{tabular}

difluoroethylenes. In fact, this transition is forbidden in the centrosymmetric difluoroethylene. The larger intensity for the $\pi-3 p \pi$ transition in 1,1-difluoroethylene might be due to its mixing with the strong $\pi-\pi^{*}$ excitation. This must be possible since both the states have the same symmetry in this difluoroethylene; however, it is not the case in other two fluoroethylenes. An interesting point is worth to be noticed here: Verhaart and Brongersma ${ }^{5}$ found an excitation at $8.4 \mathrm{eV}$ for this difluoroethylene in their electron-impact study and they tentatively assigned it as due to $\pi \rightarrow \sigma^{*}$ excitation. It is seen in the present study that though the excitation at $8.58 \mathrm{eV}$ is mainly due to $\pi-3 d \sigma$ configuration, the optically forbidden $\pi-\sigma^{*}$ configuration also mixes with this configuration. The calculated triplet $\pi$ - $\pi^{*}$ excitation energy (Table VIII) shows a very good agreement with the experimental values like in the other fluoroethylenes.

\section{Trifluoroethylene}

Calculations for the trifluoroethylene were done within $C_{S}$ symmetry, with the molecule placed in the $x y$ plane. The excitation energies and oscillator strengths obtained using SAC-CI calculations were summarized in Table IX along with the available experimental values. The calculations re-

TABLE VI. Several lowest-lying triplet excited states of cisdifluoroethylene obtained using SAC-CI at experimental geometry (Ref. 31). Excitation energies $(\Delta E)$.

\begin{tabular}{lccc}
\hline \hline State & Nature & $\begin{array}{c}\text { SAC-CI } \\
\Delta E(\mathrm{eV})\end{array}$ & Expt. \\
\hline $1{ }^{3} B_{2}$ & $\pi-\pi^{*}$ & 4.55 & $4.43^{\mathrm{a}}$ \\
$1{ }^{3} B_{1}$ & $\pi-3 s$ & 6.42 & \\
$2{ }^{3} B_{1}$ & $\pi-3 p \sigma_{z} / \sigma^{*}$ & 7.28 & \\
$1{ }^{3} A_{1}$ & $\pi-3 p \pi$ & 7.96 & \\
$1{ }^{3} A_{2}$ & $\pi-3 p \sigma_{y}$ & 8.02 & \\
$2{ }^{3} A_{2}$ & $\pi-3 d \sigma$ & 8.86 & \\
$2{ }^{3} A_{1}$ & $\pi-3 d \pi$ & 8.97 & \\
$2{ }^{3} B_{2}$ & $\pi-3 d \pi$ & 8.99 & \\
\hline
\end{tabular}

${ }^{\mathrm{a}}$ Reference 4. veal that there are lots of mixing between the states. Since this molecule has a low symmetry, $C_{s}$, and different states exist within a closed energy range, such mixings between the states are expected. Especially, the mixing between $2{ }^{1} A^{\prime}$ and $3{ }^{1} A^{\prime}$ states, the states with largest oscillator strengths, is very strong.

Unlike in the other fluoroethylenes, the excitation energy value $(8.43 \mathrm{eV})$ calculated for the main transition $\left(\pi \rightarrow \pi^{*}\right.$, $3{ }^{1} A^{\prime}$ state) in trifluoroethylene has a considerable difference with the experimental values $(7.61-7.75 \mathrm{eV})$ obtained for the strongest absorption peak. Careful analysis reveals that this state has both $\pi-\pi^{*}$ and $\pi-3 p \pi$ configurations. A small $\pi$ - $3 d \pi$ contribution was also found in the $3{ }^{1} A^{\prime}$ state. The strong mixing of $\pi-3 p \pi$ configuration with the main $\pi-\pi^{*}$ configuration might be a possible reason, at least partially, for the large difference noticed between the calculated and the experimental values. It should be mentioned here that the $2{ }^{1} A^{\prime}$ state with $7.79 \mathrm{eV}$ excitation energy also has both $\pi-\pi^{*}$ and $\pi-3 p \pi$ configurations. The calculated second moments, $\left\langle r^{2}\right\rangle$, for these two states, $3{ }^{1} A^{\prime}$ and $2{ }^{1} A^{\prime}$, are nearly equal. Because of the strong mixing, the $2{ }^{1} A^{\prime}$ state also has a large oscillator strength. It is possible that the near degeneracy of the $\pi \rightarrow \pi^{*}$ and $\pi \rightarrow 3 p \pi$ excitations gives the latter a large intensity through configuration interaction, or it may be really a part of the $\pi-\pi^{*}$ band rather than the $\pi-3 p \pi$ excitation band. It should also be noticed here that among all the fluoroethylenes, the strongest $\pi \rightarrow 3 p$ excitation is found in trifluoroethylene. ${ }^{1}$ All these points support our earlier argument that the mixing between $\pi-\pi^{*}$ and $\pi-3 p \pi$ configurations is very strong which could be a partial reason for the large difference found between the calculated and the experimental values for the main excitation. Apart from this, the reliability of the experimental values is also a concern which would be discussed in the next subsection. And, in fact, this strong mixing makes it difficult to distinguish these two states clearly.

Table IX shows that the excitation energies obtained for all other transitions agree well with the experimental values. Interestingly, the $2{ }^{1} A^{\prime \prime}$ state has a small contribution of $\pi$ 
TABLE VII. Several lowest-lying singlet excited states of 1,1-diffuoroethylene obtained using SAC-CI at experimental geometry (Ref. 32). Excitation energies $(\Delta E)$, oscillator strengths $(f)$, and second moments $\left(\left\langle r^{2}\right\rangle\right)$.

\begin{tabular}{|c|c|c|c|c|c|}
\hline \multirow[b]{2}{*}{ State } & \multirow[b]{2}{*}{ Nature } & \multicolumn{3}{|c|}{ SAC-CI } & \multirow[b]{2}{*}{ Expt. } \\
\hline & & $\Delta E(\mathrm{eV})$ & $f$ & $\left\langle r^{2}\right\rangle$ & \\
\hline $1^{1} A_{1}$ & G.S. & & & 229.9 & \\
\hline $1{ }^{1} B_{1}$ & $\pi-3 s$ & 7.05 & 0.0580 & 265.1 & $\begin{array}{c}6.82,,^{\mathrm{a}} 6.74,{ }^{\mathrm{b}} 6.95{ }^{\mathrm{c}}{ }^{\circ} \\
6.78^{\mathrm{d}}\end{array}$ \\
\hline $2{ }^{1} A_{1}$ & $\pi-\pi^{*} / 3 p \pi$ & 7.74 & 0.2470 & 262.3 & $7.50,^{\mathrm{a}, \mathrm{c}} 7.51,{ }^{\mathrm{b}} 7.60^{\mathrm{e}}$ \\
\hline $1{ }^{1} A_{2}$ & $\pi-3 p \sigma_{y}$ & 8.02 & $\cdots$ & 290.4 & \\
\hline $2{ }^{1} B_{1}$ & $\pi-3 p \sigma_{z}$ & 8.06 & 0.0009 & 295.9 & \\
\hline $3{ }^{1} A_{1}$ & $\pi-3 p \pi / \pi^{*} / 3 d \pi$ & 8.58 & 0.1588 & 287.4 & $8.22,{ }^{\mathrm{a}} 7.91,{ }^{\mathrm{b}} 8.23^{\mathrm{c}}$ \\
\hline $2{ }^{1} A_{2}$ & $\pi-3 d \sigma / \sigma^{*}$ & 8.58 & $\ldots$ & 288.8 & $8.40 ?^{\mathrm{d}}$ \\
\hline $3{ }^{1} B_{1}$ & $\pi-3 d \sigma$ & 8.84 & 0.0023 & 323.9 & \\
\hline${ }^{1} B_{2}$ & $\pi-3 d \pi$ & 8.93 & 0.0119 & 339.2 & \\
\hline $4{ }^{1} B_{1}$ & $\pi-3 d \sigma$ & 8.93 & 0.0431 & 338.5 & $8.78,^{b} 9.26^{c}$ \\
\hline $4{ }^{1} A_{1}$ & $\pi-3 d \pi / 3 p \pi$ & 9.11 & 0.0404 & 384.9 & \\
\hline
\end{tabular}

${ }^{\mathrm{a}}$ Reference 7 .

${ }^{\mathrm{b}}$ Reference 1.

${ }^{\mathrm{c}}$ Reference 4 .

${ }^{\mathrm{d}}$ Reference 5 .

${ }^{\mathrm{e}}$ Reference 33.

$-\sigma^{*}$ state. This supports the following two results. (1) Dauber and Brith $^{3}$ noticed in their study that an additional transition is observed in the spectra of trifluoroethylene, to the lower energy side of the $\pi \rightarrow \pi^{*}$ transition. But the band was very weak and was observed clearly only in the solid phase and in the matrix. They also mentioned that this transition is probably responsible for the weak shoulder at $178 \mathrm{~nm}(6.97 \mathrm{eV})$ in gaseous trifluoroethylene. (2) With the support from the speculation made by Robin, ${ }^{7}$ a weak singlet transition observed at $7.1 \mathrm{eV}$ in the study of Coggiola et $\mathrm{ll}^{4}{ }^{4}$ was considered as the $\pi \rightarrow \sigma^{*}$ valence-shell excitation. The results obtained for the triplet excitations were tabulated in Table X. Only one experimental value is available for comparison and again the calculated value agrees well with the experimental value.

\section{Tetrafluoroethylene}

Tetrafluoroethylene has $D_{2 h}$ symmetry. The electronic spectrum was obtained by keeping this symmetry, with the

TABLE VIII. Several lowest-lying triplet excited states of 1,1difluoroethylene obtained using SAC-CI at experimental geometry (Ref 32). Excitations energies $(\Delta E)$.

\begin{tabular}{cccc}
\hline \hline State & Nature & $\begin{array}{c}\text { SAC-CI } \\
\Delta E(\mathrm{eV})\end{array}$ & Expt. \\
\hline $1{ }^{3} A_{1}$ & $\pi-\pi^{*}$ & 4.57 & $4.60,{ }^{\mathrm{a}} 4.63,{ }^{\mathrm{b}} 4.70^{\mathrm{c}}$ \\
$1{ }^{3} B_{1}$ & $\pi-3 s$ & 6.85 & $6.60^{\mathrm{c}}$ \\
$1{ }^{3} A_{2}$ & $\pi-3 p \sigma_{y}$ & 8.00 & \\
$2{ }^{3} B_{1}$ & $\pi-3 p \sigma_{z}$ & 8.01 & \\
$2{ }^{3} A_{1}$ & $\pi-3 p \pi$ & 8.08 & \\
$2{ }^{3} A_{2}$ & $\pi-\sigma^{*} / 3 d \sigma$ & 8.52 & \\
$3{ }^{3} B_{1}$ & $\pi-3 d \sigma$ & 8.80 & \\
$1{ }^{3} B_{2}$ & $\pi-3 d \pi$ & 8.93 & \\
$3{ }^{3} A_{1}$ & $\pi-3 d \pi$ & 9.01 & \\
\hline \hline
\end{tabular}

${ }^{\mathrm{a}}$ Reference 33 .

${ }^{\mathrm{b}}$ Reference 4.

${ }^{\mathrm{c}}$ Reference 5 . molecule placed in the $y z$ plane where $z$ axis is the molecular axis. Table XI shows the SAC-CI excitation energies and oscillator strengths obtained for this fluoroethylene with the available experimental results. Winstead and $\mathrm{McKoy}^{35}$ calculated cross sections for elastic and inelastic collisions of lowenergy electrons with tetrafluoroethylene. The authors also calculated excitation energies of several states by using single-excitation configuration-interaction (SECI) theory with $6-311+\mathrm{G}(3 d)$ basis set. These values were also tabulated in Table XI.

The main excitation once again is the $\pi \rightarrow \pi^{*}$ valence transition. The calculated excitation energy $(9.41 \mathrm{eV})$ for this transition is around $0.4 \mathrm{eV}$ larger than the value obtained in a recent experimental study $(9.02 \mathrm{eV}){ }^{6}$ A small mixing of $\pi$ $-3 d \pi$ configuration with the $\pi-\pi^{*}$ state is noticed. Excitation energy obtained for the first Rydberg state, $\pi-3 s$, differs moderately from the experimental values. One point worth to be noticed here is that this Rydberg state is mixed slightly with a bright, optically allowed, $\pi-\sigma^{*}$ state which has the same $B_{3 u}$ symmetry as the Rydberg $\pi-3 s$ state has. This $\pi$ $\rightarrow \sigma^{*}$ excitation mixing could significantly perturb the $\pi$ $\rightarrow 3 s$ excitation. A weak peak found in the earlier experimental study at around $7.7 \mathrm{eV}$ was considered as due to $\pi-\sigma^{*}$ excitation; ${ }^{4,6,7}$ however, such a transition was not found separately in the present study. On the other hand, as mentioned above, the $1{ }^{1} B_{3 u} \pi$-3s state has a small admixture of $\pi \rightarrow \sigma^{*}$ transition with the same $B_{3 u}$ symmetry. The $1{ }^{1} B_{1 u}$ $\pi$ - $3 d \pi$ state has a small contribution from $\pi \rightarrow \pi^{*}$ transition. The experimental peak of this feature is diffuse and the experimental energy position was noticed to be subject to great uncertain $^{6}$ and hence the energy difference between the SAC-CI and the experimental value seen for this transition (Table XI) is need not to be considered seriously. For all the other states, the calculated values agree well with the experimental values. The calculated triplet excitation energy values were tabulated in Table XII. 
TABLE IX. Several lowest-lying singlet excited states of trifluoroethylene obtained using SAC-CI at B3LYP/ aug-cc-pVTZ geometry. Excitation energies $(\Delta E)$, oscillator strengths $(f)$, and second moments $\left(\left\langle r^{2}\right\rangle\right)$.

\begin{tabular}{lccccc}
\hline \hline & & \multicolumn{3}{c}{ SAC-CI } \\
\cline { 3 - 4 } State & Nature & $\Delta E(\mathrm{eV})$ & $f$ & $\left\langle r^{2}\right\rangle$ & \multirow{2}{*}{ Expt. } \\
\hline $1^{1} A^{\prime}$ & G. S. & & & 371.4 & \\
$1{ }^{1} A^{\prime \prime}$ & $\pi-3 s$ & 6.65 & 0.0287 & 391.7 & $6.50,{ }^{\mathrm{a}, \mathrm{b}} 6.56^{\mathrm{c}}$ \\
$2{ }^{1} A^{\prime \prime}$ & $\pi-3 p \sigma_{y} / 3 s / \sigma^{*}$ & 7.23 & 0.0120 & 398.9 & $7.1,{ }^{\mathrm{c}} 6.97^{\mathrm{d}}$ \\
$2{ }^{1} A^{\prime}$ & $\pi-3 p \pi+\pi-\pi^{*}$ & 7.79 & 0.1151 & 416.2 & $7.61,{ }^{\mathrm{a}} 7.75,{ }^{\mathrm{b}} 7.65^{\mathrm{c}}$ \\
$3{ }^{1} A^{\prime \prime}$ & $\pi-3 p \sigma_{x}$ & 7.86 & 0.0028 & 427.7 & $7.97,,^{\mathrm{a}, \mathrm{b}} 7.98^{\mathrm{c}}$ \\
$3{ }^{1} A^{\prime}$ & $\pi-\pi^{*}+\pi-3 p \pi / 3 d \pi$ & 8.43 & 0.2117 & 416.2 & $7.61,{ }^{\mathrm{a}} 7.75,{ }^{\mathrm{b}} 7.65^{\mathrm{c}}$ \\
$5{ }^{1} A^{\prime \prime}$ & $\pi-3 d \sigma$ & 8.79 & 0.0286 & 491.8 & \\
$4{ }^{1} A^{\prime}$ & $\pi-3 d \pi$ & 8.88 & 0.0004 & 500.6 & \\
$6{ }^{1} A^{\prime \prime}$ & $\pi-3 d \sigma$ & 8.89 & 0.0000 & 502.3 & \\
$7{ }^{1} A^{\prime \prime}$ & $\pi-3 d \sigma$ & 8.94 & 0.0009 & 520.0 & \\
$5{ }^{1} A^{\prime}$ & $\pi-3 d \pi$ & 8.99 & 0.0853 & 513.0 & $8.71,,^{\mathrm{b}} 8.91^{\mathrm{c}}$ \\
\hline \hline
\end{tabular}

${ }^{\mathrm{a}}$ Reference 7 .

${ }^{\mathrm{b}}$ Reference 1.

${ }^{\mathrm{c}}$ Reference 4.

${ }^{\mathrm{d}}$ Reference 3 .

Although the calculated excitation energies for different states in all the fluoroethylenes agree well with the experimental values, there are cases where the difference of more than $0.3 \mathrm{eV}$ is noticed; for example, the $\pi-\pi^{*}$ states in trifluoroethylene and tetrafluoroethylene. This needs explanations and accordingly the possible sources for the differences are discussed here. Among the many possible reasons, the first and the foremost is the applicability of the theory used. Now it is generally recognized that the SAC-CI is an established theory to obtain the accurate excitation energies. Even for many problematic transitions in certain molecules, the results derived using SAC-CI theory were proven to be very accurate. One such example is the $\pi$ - $\pi^{*}$ state in cyclopentadiene. It has been shown recently that the vertical excitation energy obtained for this state using SAC-CI is the most reliable among the other values. ${ }^{36}$ Our earlier study on vinyl chloride has also shown that the excitation energies calculated using SAC-CI are more accurate than those obtained using MRCI theory. ${ }^{28}$ Even here it is noticed that the SAC-CI theory provides highly accurate results for vinyl fluoride (Table I). So we are confident that the SAC-CI theory is not the source for the differences noticed, in a few cases, be-

TABLE X. Several lowest-lying triplet excited states of trifluoroethylene obtained using SAC-CI at B3LYP/aug-cc-pVTZ geometry. Excitation energies $(\Delta E)$.

\begin{tabular}{lccc}
\hline \hline State & & SAC-CI & \\
\hline $1{ }^{3} A^{\prime}$ & Nature & $\Delta E(\mathrm{eV})$ & Expt. \\
$1^{3} A^{\prime \prime}$ & $\pi-\pi^{*}$ & 4.63 & $4.43^{\mathrm{a}}$ \\
$2{ }^{3} A^{\prime \prime}$ & $\pi-3 s$ & 6.42 & \\
$3{ }^{3} A^{\prime \prime}$ & $\pi-3 p \sigma_{y} / 3 s / \sigma^{*}$ & 7.03 & \\
$2{ }^{3} A^{\prime}$ & $\pi-3 p \sigma_{x}$ & 7.81 & \\
$5^{3} A^{\prime \prime}$ & $\pi-3 p \pi$ & 7.91 & \\
$3{ }^{3} A^{\prime}$ & $\pi-3 d \sigma$ & 8.74 & \\
$4^{3} A^{\prime}$ & $\pi-3 d \pi$ & 8.86 & \\
\hline
\end{tabular}

${ }^{\mathrm{a}}$ Reference 4 . tween the calculated and the experimental values. The next possible reason might be the insufficiency of basis set utilized. We used aug-cc-pVTZ basis set incorporating two sets of Rydberg spd functions for all the heavy atoms in all the calculations. In any sense, this basis set must be sufficient enough to study the excited states. The same basis set combinations provided excellent results for ethylene and vinyl chloride. ${ }^{28}$ The results obtained for vinyl fluoride using the same basis set combination in the present study are shown to be very accurate. So we believe that the basis set used for the calculations is sufficient.

It is understandable, however, that though the level of theory used for the calculations are adequate, the calculated excitation energies would improve slightly if the effects of vibrational structure and the nonadiabatic coupling are incorporated in the calculations. On the other hand, it is possible that the absorption maxima for some excitations, especially for $\pi \rightarrow \pi^{*}$ transitions in some cases, may not be representing the vertical excitation energy as it has been seen in many cases including in ethylene. ${ }^{36-38}$ In addition to that the experimental $\pi-\pi^{*}$ absorption bands in some fluoroethylenes are very broad and they are superimposed with many Rydberg states. Foregoing arguments support that one does not need to believe that the absorption maxima obtained in the experimental studies are accurately representing the vertical excitation energies in all the cases. Furthermore, there is also some strong mixing between near-degenerate $\pi-\pi^{*}$ and Rydberg states in many fluoroethylenes. Apart from all these facts, it is worth mentioning here that almost all the experimental absorption spectra were obtained at least 30 years before. By considering the fact that a difference of about $0.2 \mathrm{eV}$ is found in between the early ${ }^{4}$ and the recently ${ }^{6}$ obtained experimental values for the $\pi$ - $\pi^{*}$ excitation energies in tetrafluoroethylene, there is a room to believe that the available experimental values are not free from uncertainty. 
TABLE XI. Several lowest-lying singlet excited states of tetrafluoroethylene obtained using SAC-CI at experimental geometry (Ref. 34). Excitation energies $(\Delta E)$, oscillator strengths $(f)$, and second moments $\left(\left\langle r^{2}\right\rangle\right)$.

\begin{tabular}{|c|c|c|c|c|c|c|}
\hline \multirow[b]{2}{*}{ State } & \multirow[b]{2}{*}{ Nature } & \multicolumn{3}{|c|}{ SAC-CI } & \multicolumn{2}{|c|}{ Past studies } \\
\hline & & $\Delta E(\mathrm{eV})$ & $f$ & $\left\langle r^{2}\right\rangle$ & $\mathrm{SECI}^{\mathrm{a}}$ & Expt. \\
\hline $1{ }^{1} A_{g}$ & G.S. & & & 491.3 & & \\
\hline $1{ }^{1} B_{1 g}^{8}$ & $\pi-\sigma^{*}$ & 7.01 & $\ldots$ & 497.7 & 7.55 & \\
\hline $1{ }^{1} B_{3 u}$ & $\pi-3 s / \sigma^{*}$ & 7.09 & 0.0381 & 517.8 & 7.74 & $\begin{array}{c}6.55,^{\mathrm{b}} 6.37,^{\mathrm{c}} \\
6.62,{ }^{\mathrm{d}} 6.40^{\mathrm{e}}\end{array}$ \\
\hline $1{ }^{1} B_{2 g}$ & $\pi-3 p \sigma_{z}$ & 8.24 & $\cdots$ & 545.5 & 9.21 & $8.02{ }^{b}{ }^{b} 8.01^{c-e}$ \\
\hline $2{ }^{1} A_{g}$ & $\pi-3 p \pi$ & 8.24 & $\cdots$ & 548.5 & 9.21 & \\
\hline $2{ }^{1} B_{1 g}^{g}$ & $\pi-3 p \sigma_{y}$ & 8.76 & $\cdots$ & 582.7 & & \\
\hline $2{ }^{1} B_{3 u}$ & $\pi-3 d \sigma$ & 9.03 & 0.0254 & 619.2 & & $8.64^{\mathrm{c}, \mathrm{e}} 9.04^{\mathrm{d}}$ \\
\hline $1{ }^{1} B_{1 u}$ & $\pi-3 d \pi / \pi^{*}$ & 9.19 & 0.0698 & 593.5 & & $8.39^{\mathrm{e}}$ \\
\hline $1{ }^{1} B_{2 u}$ & $\pi-3 d \pi$ & 9.22 & 0.0001 & 610.8 & & \\
\hline $3{ }^{1} B_{3 u}$ & $\pi-3 d \sigma$ & 9.29 & 0.0000 & 628.0 & & \\
\hline $1{ }^{1} A_{u}$ & $\pi-3 d \sigma$ & 9.30 & $\ldots$ & 644.6 & & \\
\hline $2{ }^{1} B_{1 u}$ & $\pi-\pi^{*}$ & 9.41 & 0.5108 & 520.9 & 9.71 & $\begin{array}{l}8.88,{ }^{\mathrm{b}} 8.89,^{\mathrm{c}} \\
8.844^{\mathrm{d}} 9.02^{\mathrm{e}}\end{array}$ \\
\hline
\end{tabular}

${ }^{\mathrm{a}}$ Reference 35 .

${ }^{\mathrm{b}}$ Reference 7 .

${ }^{\mathrm{c}}$ Reference 1 .

${ }^{\mathrm{d}}$ Reference 4 .

${ }^{\mathrm{e}}$ Reference 6 .

It should also be mentioned here that noticeable differences are found even among a few other experimental values too (see Tables).

It is found in the present study that all the excitations of the fluoroethylenes in the investigated energy region are from HOMO $\pi$ orbitals. The fluorine nonbonding orbitals are deeply bound due to the high electronegativity of the fluorine atoms and hence no excitation from these $n$ orbitals was found in the energy region studied in the present investigation.

\section{E. The $\pi \rightarrow \pi^{*}$ blueshift}

As mentioned in the Introduction, an interesting fact about the fluoroethylenes is the blueshift of around $1.25 \mathrm{eV}$

TABLE XII. Several lowest-lying triplet excited states of tetrafluoroethylene obtained using SAC-CI at experimental geometry (Ref. 34). Excitation energies $(\Delta E)$.

\begin{tabular}{ccccc}
\hline \hline & & & \multicolumn{2}{c}{ Past studies } \\
\cline { 3 - 5 } State & Nature & $\begin{array}{c}\text { SAC-CI } \\
\Delta E(\mathrm{eV})\end{array}$ & SECI $^{\mathrm{a}}$ & Expt. \\
\hline $1{ }^{3} B_{1 u}$ & $\pi-\pi^{*}$ & 5.12 & 4.48 & $4.68,{ }^{\mathrm{b}} 4.79^{\mathrm{c}}$ \\
$1{ }^{3} B_{1 g}$ & $\pi-\sigma^{*}$ & 6.69 & 6.76 & \\
$1{ }^{3} B_{3 u}$ & $\pi-3 s$ & 6.81 & 7.17 & \\
$1{ }^{3} B_{2 g}$ & $\pi-3 p \sigma_{z}$ & 8.07 & 8.55 & \\
$1{ }^{3} A_{g}$ & $\pi-3 p \pi$ & 8.20 & 8.95 & \\
$2{ }^{3} B_{1 g}$ & $\pi-3 p \sigma_{y}$ & 8.73 & & \\
$2{ }^{3} B_{3 u}$ & $\pi-3 d \sigma$ & 8.93 & & \\
$2{ }^{3} B_{1 u}$ & $\pi-3 d \pi$ & 9.22 & & \\
$1{ }^{3} B_{2 u}$ & $\pi-3 d \pi$ & 9.22 & & \\
$3{ }^{3} B_{3 u}$ & $\pi-3 d \sigma$ & 9.29 & & \\
$1{ }^{3} A_{u}$ & $\pi-3 d \sigma$ & 9.31 & & \\
\hline \hline
\end{tabular}

${ }^{\mathrm{a}}$ Reference 35 .

${ }^{\mathrm{b}}$ Reference 4.

${ }^{\mathrm{c}}$ Reference 6. in the $\pi \rightarrow \pi^{*}$ excitation energy of tetrafluoroethylene from that of trifluoroethylene. A similar kind of blueshift trend is also seen in the present study. Resistance to torsional motion in the $\pi^{*}$ due to the substitution of the final hydrogen was previously invoked to explain the large $\pi \rightarrow \pi^{*}$ excitation energy shift in the tetrafluoroethylene. To test this idea, Coggiola et $a .^{4}$ studied the chlorotrifluoroethylene using electron-impact spectroscopy. In this molecule, the last hydrogen is replaced by chlorine which should constitute an even greater hindrance for torsional motion of the molecule. The result, however, showed no appreciable shift in the $\pi$ $\rightarrow \pi^{*}$ transition energy produced by the replacement of the hydrogen in trifluoroethylene with chlorine. Therefore it has been concluded that the large shift in the $\pi \rightarrow \pi^{*}$ transition energy of tetrafluoroethylene is presumably not due to steric effects but may be due to electronic effects.

Intermeidate neglect of differential overlap-CI calculations have shown that in all the fluoroethylenes a $\sigma-\sigma^{*}$ configuration exists above the $\pi-\pi^{*}$ configuration. ${ }^{39}$ It has been argued that the interaction between these two configurations lowers the energy of the $\pi^{*}$ level considerably. ${ }^{39}$ In tetrafluoroethylene, however, this interaction cannot take place since the $\sigma-\sigma^{*}$ level is of much higher energy which reflects in the blueshift in the $\pi \rightarrow \pi^{*}$ excitation energy of the tetrafluoroethylene. That is, in the partially fluorinated ethylenes, the $\sigma_{(\mathrm{C}-\mathrm{C}-\mathrm{H})} \rightarrow \sigma^{*}$ configurations interact with the $\pi \rightarrow \pi^{*}$ configurations but in the tetrafluoroethylene, there are no $\mathrm{C}-\mathrm{H}$ bonds and the $\sigma_{(\mathrm{C}-\mathrm{C}-\mathrm{F})}^{*}$ states come at much higher energies resulting to less or no interaction with the $\pi \rightarrow \pi^{*}$ configuration.

This could be checked in the present study. Thorough analysis of the nature of the $\pi-\pi^{*}$ excitations in all the fluoroethylenes reveals that these states are completely dominated by the transitions from the $\pi$ HOMO orbitals. The 
contribution of $\sigma-\sigma^{*}$ configuration in these $\pi-\pi^{*}$ states is negligible (less than 0.03). As Tables I-XII show, no $\sigma$ $\rightarrow \sigma^{*}$ transition in any of the fluoroethylenes was identified in the energy region investigated in the present study. To find the existence of $\sigma-\sigma^{*}$ excited state in the extended energy region, we studied several more singlet excited states of vinyl fluoride in which the fluorine substitution stabilizes the $\sigma$ orbital less than it does in the other fluoroethylenes. For this purpose, 20 lowest-lying singlet states of vinyl fluoride were investigated.

Results reveal that 3 states among the 20 states studied with the $A^{\prime}$ symmetry, the same symmetry which the $\sigma$ - $\sigma^{*}$ state possesses, are due to the transitions from $\sigma$ states. These are Rydberg $13{ }^{1} A^{\prime} \sigma-3 s(10.71 \mathrm{eV}), \quad 17{ }^{1} A^{\prime} \sigma$ $-3 p \sigma_{x}(11.42 \mathrm{eV})$, and $19{ }^{1} A^{\prime} \sigma-3 p \sigma_{y}(11.55 \mathrm{eV})$ excited states. No valence $\sigma-\sigma^{*}$ excited state was found in this energy region (up to $12 \mathrm{eV}$ ) too. By considering the fact that the excitation energy found for the $\pi \rightarrow \pi^{*}$ transition in vinyl fluoride at the same level of theory is $7.68 \mathrm{eV}$, the energy gap between the so-called interacting $\pi-\pi^{*}$ and $\sigma-\sigma^{*}$ states must be more than $4 \mathrm{eV}$. It is known that fluorination (ethylene to tetrafluoroethylene) stabilizes the $\pi$ MOs by around $0.2 \mathrm{eV}$ and the $\sigma$ MOs by $2.5-4 \mathrm{eV} .{ }^{20}$ Taking into consideration that the energy gap between the $\pi-\pi^{*}$ and $\sigma-\sigma^{*}$ states in vinyl fluoride would be expected to be greater than $4 \mathrm{eV}$ and the $\sigma$ orbital in trifluoroethylene is stabilized roughly around $1 \mathrm{eV}$ more than that in vinyl fluoride, ${ }^{20}$ the energy gap between the probable interacting $\pi-\pi^{*}$ and $\sigma-\sigma^{*}$ states in trifluoroethylene is expected to be greater than $5 \mathrm{eV}$ ! In such a scenario, the interaction between $\pi-\pi^{*}$ and $\sigma-\sigma^{*}$ excited states should be negligible. So the existence of the $\sigma-\sigma^{*}$ state above the $\pi-\pi^{*}$ state might not be a possible reason for the blueshift of around $1.25 \mathrm{eV}$ found in the $\pi \rightarrow \pi^{*}$ transition energy in tetrafluoroethylene.

Earlier, electron transmission spectroscopy was employed to measure the gas phase electron affinities of the fluoroethylenes. ${ }^{40}$ Authors of that study showed that while inductive and resonance effects alone could not explain the trends in the electron affinities, consideration of geometrical changes together with these interactions is important to explain the trends qualitatively. The same consideration could be used here to explain the $\pi-\pi^{*}$ blueshift. A standout truth in tetrafluoroethylene from that in the other fluoroethylenes is the absence of $\mathrm{H}$ atom that must reflect in the structure. The interesting fact is that both $\mathrm{C}-\mathrm{F}$ and $\mathrm{C}=\mathrm{C}$ bond lengths in tetrafluoroethylene are shorter than those in the other fluoroethylenes (Table XIII). Reduction in $\mathrm{C}=\mathrm{C}$ bond length should stabilize the $\pi$ orbitals and destabilize the $\pi^{*}$ orbitals and on the other hand, reduction in the $\mathrm{C}-\mathrm{F}$ bond length can destabilize both $\pi$ and $\pi^{*}$ orbitals since the fluorine $p_{z}$ orbital which is perpendicular to the plane of the molecule interacts in an antibonding manner in both cases. Thus, these effects are additive and destabilizing for the $\pi^{*}$ orbital. Our preliminary calculations support this phenomenon: while the difference in $\pi^{*}$ orbital energies is very small among fluoroethylenes up to trifluoroethylene, the same $\pi^{*}$ orbital energy in tetrafluoroethylene, however, is about $0.6 \mathrm{eV}$ higher than that in other fluoroethylenes. So it is conceivable that the com-
TABLE XIII. Experimental $\mathrm{C}=\mathrm{C}$ and $\mathrm{C}-\mathrm{F}$ bond lengths. Bond lengths $(R)$ are in angstroms.

\begin{tabular}{ccc}
\hline \hline Molecule & $R(\mathrm{C}-\mathrm{F})$ & $R(\mathrm{C}=\mathrm{C})$ \\
\hline $\mathrm{C}_{2} \mathrm{H}_{4}$ & - & $1.339^{\mathrm{a}}$ \\
$\mathrm{C}_{2} \mathrm{H}_{3} \mathrm{~F}$ & $1.346^{\mathrm{b}}$ & $1.329^{\mathrm{b}}$ \\
trans $-\mathrm{C}_{2} \mathrm{H}_{2} \mathrm{~F}_{2}$ & $1.352^{\mathrm{c}}$ & $1.316^{\mathrm{c}}$ \\
cis $-\mathrm{C}_{2} \mathrm{H}_{2} \mathrm{~F}_{2}$ & $1.337^{\mathrm{d}}$ & $1.325^{\mathrm{d}}$ \\
$1,1-\mathrm{C}_{2} \mathrm{H}_{2} \mathrm{~F}_{2}$ & $1.315^{\mathrm{e}}$ & $1.340^{\mathrm{e}}$ \\
$\mathrm{C}_{2} \mathrm{HF}_{3}$ & $1.316 / 1.342^{\mathrm{f}}$ & $1.341^{\mathrm{f}}$ \\
$\mathrm{C}_{2} \mathrm{~F}_{4}$ & $1.319^{\mathrm{g}}$ & $1.311^{\mathrm{g}}$ \\
\hline \hline
\end{tabular}

${ }^{\mathrm{a}}$ Reference 41 .

${ }^{\mathrm{b}}$ Reference 29 .

${ }^{\mathrm{c}}$ Reference 30.

${ }^{\mathrm{d}}$ Reference 31 .

${ }^{\mathrm{e}}$ Reference 32.

${ }^{\mathrm{f}}$ Reference 42.

${ }^{\mathrm{g}}$ Reference 34 .

bined $\mathrm{C}-\mathrm{F}$ and $\mathrm{C}=\mathrm{C}$ bond shrinkages in tetrafluoroethylene might be a possible reason for the blueshift in the $\pi \rightarrow \pi^{*}$ excitation energy of this molecule.

\section{CONCLUDING REMARKS}

The electronic spectra of all the six fluorine substituted ethylenes, mono- to tetrafluoroethylene, were studied using SAC-CI theory with large basis set incorporated with the necessary Rydberg spd functions.

The vertical excitation energies obtained for different states in all the fluoroethylenes agree very well with the available experimental values in almost all the cases except for the notorious $\pi \rightarrow \pi^{*}$ transitions in a few fluoroethylenes. Even in this challenging $\pi \rightarrow \pi^{*}$ excitations, the values obtained in the present study agree better with the available experimental values than those calculated in the other theoretical studies. Strong mixing between the valence $\pi-\pi^{*}$ and the Rydberg $\pi-3 p \pi$ states in trifluoroethylene makes the assignment somehow difficult. Because of this mixing, the $\pi$ $-3 p \pi$ state in this fluoroethylene has large oscillator strength. Though a few Rydberg states in some fluoroethylenes have the admixture of $\pi \rightarrow \sigma^{*}$ transitions, none of the excitations in all the fluoroethylenes, except a dark $1{ }^{1} B_{1 g} \pi$ - $\sigma^{*}$ state in tetrafluoroethylene, has pure $\pi \rightarrow \sigma^{*}$ valence character. With the fluorine nonbonding orbitals lying at very low energies, the present study reveals that all the excitations of the fluoroethylenes in the studied energy region are from HOMO $\pi$ orbitals. The present study helped us to clarify some of the speculative assignments made in the experimental studies.

The interaction between the $\pi-\pi^{*}$ and $\sigma-\sigma^{*}$ configurations has been believed to be the reason behind the $1.25 \mathrm{eV}$ blueshift in the $\pi \rightarrow \pi^{*}$ excitation energy of tetrafluoroethylene from that of trifluoroethylene. However, no $\sigma \rightarrow \sigma^{*}$ transition is identified in the energy region investigated in the present study. Further extended study on vinyl fluoride showed that no $\sigma-\sigma^{*}$ state exists below $12 \mathrm{eV}$ energy range. With these results, analysis reveals that the energy difference between the $\pi-\pi^{*}$ and $\sigma-\sigma^{*}$ states in trifluoroethylene would be expected to be larger than $5 \mathrm{eV}$. The large energy gap shows that the existence of $\sigma-\sigma^{*}$ state above the $\pi-\pi^{*}$ state may not be the reason for the blueshift. The shrinkage of the 
$\mathrm{C}=\mathrm{C}$ and $\mathrm{C}-\mathrm{F}$ bonds in tetrafluoroethylene might be a possible reason for this blueshift, at least partially.

\section{ACKNOWLEDGMENT}

This study has been supported by the Grant for Creative Scientific Research from the Ministry of Education, Science, Sports, and Culture of Japan.

${ }^{1}$ G. Belanger and C. Sandorfy, J. Chem. Phys. 55, 2055 (1971).

${ }^{2}$ R. F. Lake and H. Thompson, Proc. R. Soc. London, Ser. A 315, 323 (1970).

${ }^{3}$ P. Dauber and M. Brith, Chem. Phys. 11, 143 (1975).

${ }^{4}$ M. J. Coggiola, W. M. Flicker, O. A. Mosher, and A. Kuppermann, J. Chem. Phys. 65, 2655 (1976).

${ }^{5}$ G. J. Verhaart and H. H. Brongersma, Chem. Phys. 52, 431 (1980),

${ }^{6}$ S. Eden, P. Limao-Vieira, P. A. Kendall et al., Chem. Phys. 297, 257 (2004).

${ }^{7}$ M. B. Robin, Higher Excited States of Polyatomic Molecules (Academic, New York, 1975), Vol. 2, p. 56.

${ }^{8}$ M. B. Robin, Higher Excited States of Polyatomic Molecules (Academic, New York, 1985), Vol. 3, p. 236.

${ }^{9}$ W. A. Guillory and G. H. Andrews, J. Chem. Phys. 62, 3208 (1975).

${ }^{10}$ T. K. Minton, P. Felder, R. C. Scales, and J. R. Huber, Chem. Phys. Lett. 164, 113 (1989).

${ }^{11}$ G. E. Hall, J. T. Muckerman, J. M. Preses, R. E. Weston, Jr., G. W. Flynn, and A. Persky, J. Chem. Phys. 101, 3679 (1994).

${ }^{12}$ K. Sato, S. Tsunashima, T. Takayanagi, G. Fujisawa, and A. Yokoyama, Chem. Phys. Lett. 242, 401 (1995).

${ }^{13}$ B. A. Balko, J. Zhang, and Y. T. Lee, J. Phys. Chem. A 101, 6611 (1997).

${ }^{14}$ J. J. Lin, S. M. Wu, D. W. Hwang, Y. T. Lee, and X. Yang, J. Chem. Phys. 109, 10838 (1998).

${ }^{15}$ J. J. Lin, T. C. Hsu, D. W. Hwang, Y. T. Lee, and X. Yang, J. Chem. Phys. 109, 10719 (1998).

${ }^{16}$ S.-R. Lin and Y.-P. Lee, J. Chem. Phys. 111, 9233 (1999).

${ }^{17}$ S.-R. Lin, S.-C. Lin, Y.-C. Lee, Y.-C. Chou, I.-C. Chen, and Y.-P. Lee, J. Chem. Phys. 114, 7396 (2001).

${ }^{18}$ S. A. Trushin, S. Sorgues, W. Fub, and W. E. Schmid, ChemPhysChem 5, 1389 (2004)

${ }^{19}$ Z. R. Grabowski and A. Bylina, Trans. Faraday Soc. 60, 1131 (1964).

${ }^{20}$ C. R. Brundle, M. B. Robin, N. A. Kuebler, and H. Basch, J. Am. Chem.
Soc. 94, 1451 (1972)

${ }^{21}$ H. Nakatsuji and K. Hirao, J. Chem. Phys. 68, 2053 (1978).

${ }^{22}$ H. Nakatsuji, Chem. Phys. Lett. 59, 362 (1978).

${ }^{23}$ H. Nakatsuji, Chem. Phys. Lett. 67, 329 (1979); ibid. 67, 334 (1979).

${ }^{24}$ H. Nakatsuji, Acta Chim. Hung. 129, 719 (1992); Computational Chemistry - Review of Current Trends (World Scientific, Singapore, 1997), Vol. 2, pp. 62-124.

${ }^{25}$ M. Barbatti, A. J. A. Aquino, and H. Lischka, J. Phys. Chem. A 109, 5168 (2005).

${ }^{26}$ M. J. Frisch, G. W. Trucks, H. B. Schlegel et al., Gaussian 03, Revision B.01, Gaussian, Inc., Pittsburgh, PA, 2003.

${ }^{27}$ T. H. Dunning, Jr., J. Chem. Phys. 90, 1007 (1989); D. E. Woon and T. H. Dunning, Jr., ibid. 98, 1358 (1993).

${ }^{28}$ S. Arulmozhiraja, R. Fukuda, M. Ehara, and H. Nakatsuji, J. Chem. Phys. 124, 034312 (2006).

${ }^{29}$ B. J. Smith, D. Coffey, Jr., and L. Radom, J. Chem. Phys. 97, 6113 (1992).

${ }^{30}$ N. C. Craig, O. P. Abiog, B. Hu, S. C. Stone, W. J. Lafferty, and L.-H. Xu, J. Phys. Chem. 100, 5310 (1996).

${ }^{31}$ M. D. Harmony, V. W. Laurie, R. L. Kuczkowski, R. H. Schwendeman, D. A. Ramsay, F. J. Lovas, W. J. Lafferty, and A. G. Maki, J. Phys. Chem. Ref. Data 8, 619 (1979).

${ }^{32}$ F. C. Mijlhoff, G. H. Renes, K. Kohata, K. Oyanagi, and K. Kuchitsu, J. Mol. Struct. 39, 241 (1977).

${ }^{33}$ J. H. Moore Jr., J. Phys. Chem. 76, 1130 (1972).

${ }^{34}$ J. L. Carlos, R. R. Karl, and S. H. Bauer, J. Chem. Soc., Faraday Trans. 2 70, 177 (1974)

${ }^{35}$ C. Winstead and V. McKoy, J. Chem. Phys. 116, 1380 (2002).

${ }^{36}$ Y. J. Bomble, K. W. Sattelmeyer, J. F. Stanton, and J. Gauss, J. Chem. Phys. 121, 5236 (2004).

${ }^{37}$ I. Garcia-Cuesta, A. M. J. Sanchez de Meras, and H. Koch, J. Chem. Phys. 118, 8216 (2003).

${ }^{38}$ C. Petrongolo, R. J. Buenker, and S. D. Peyerimhoff, J. Chem. Phys. 76, 3655 (1982)

${ }^{39}$ D. R. Salahub, Theor. Chim. Acta 22, 330 (1971).

${ }^{40}$ N. S. Chiu, P. D. Burrow, and K. D. Jordan, Chem. Phys. Lett. 68, 121 (1979).

${ }^{41}$ E. Hirota, Y. Endo, S. Saito, K. Yoshida, I. Yamaguchi, and K. Machida, J. Mol. Spectrosc. 89, 223 (1981).

${ }^{42}$ V. Mom, P. A. G. Huisman, F. C. Mijlhoff, and G. H. Renes, J. Mol. Struct. 62, 95 (1980) 